\title{
A Case Report of Successful Treatment of Mammary Myofibromyoblastoma in a Male Patient
}

\author{
Omer Abdalla ${ }^{\mathrm{a}, \mathrm{d}}$, Abhishek Chitnis ${ }^{\mathrm{a}}$, Banan Omer ${ }^{\mathrm{a}}$, Mohammed Elhadic, \\ Muhammad Osman Karim ${ }^{b}$, Shariq Sabri ${ }^{\mathrm{a}}$
}

\begin{abstract}
Myofibroblastoma (MFB) is an extremely rare benign tumor that can arise in various organs, but commonly in the breast. Albeit it is a benign tumor, the diagnosis is frequently challenging with insurmountable difficulties as it might be erroneously diagnosed as a malignancy. Herein we are reporting a large-sized MFB presented as a non-tender, firm mass which interestingly was adherent to the skin and was initially thought as cancer. The patient underwent a wide local excision and histology confirmed MFB, and had no evidence of recurrence up to 8 months post-operatively.
\end{abstract}

Keywords: Myofibroblastoma; Breast lump; Spindle cell tumors; Case report

\section{Introduction}

Myofibroblastoma (MFB) is an extremely rare benign tumor that can arise in various organs and tissues, but most commonly in the breast. It accounts for less than $1 \%$ of breast tumors [1]. It was first described by Wargotz et al in 1987, who reported 16 cases of this disease predominantly in men [2]. Although it is rare, it can add real challenges and difficulties to the preoperative planning and diagnosis due to its similarities to other benign and malignant breast lesions [3].

To our knowledge only a few cases were described in the literature. In this article, based on SCARE criteria we report a large-sized MFB presented as a non-tender, firm mass surprisingly adherent to the skin in the upper outer quadrant of the left breast in a male patient.

\section{Case Report}

A 45-year-old man, an otherwise fit and healthy individual,

\footnotetext{
Manuscript submitted March 9, 2018, accepted March 29, 2018

aTameside General Hospital, Ashton-under-Lyne, UK

${ }^{b}$ Buckinghamshire Healthcare NHS Trust, High Wycombe, UK

cSandwell and West Birmingham NHS Trust, Birmingham, UK

${ }^{\mathrm{d} C}$ Corresponding Author: Omer Abdalla, Tameside General Hospital, Ashtonunder-Lyne OL6 9RW, UK. Email: omer.abdalla@aol.com
}

doi: https://doi.org/10.14740/jcs340 was referred to an urgent breast clinic of a district general hospital in the UK with a 2-month history of a slowly enlarging left breast lump. Therefore, he underwent a triple assessment as per protocol.

There was no history of breast trauma, associated breast tenderness or nipple discharge. The patient had no previous history of previous breast pathology, nor any family history of breast diseases. He is not on any regular medications, a nonsmoker and denied any anabolic steroid use.

Physical examination revealed that the lump was smooth, round and well-defined, but adherent to the overlying skin. Axillary lymph nodes were not palpable and abdominal and testicular examinations were both unremarkable.

Ultrasonography examination revealed an $8 \times 3.8 \mathrm{~cm}$ round, well-defined, homogeneous mass in the upper outer quadrant of the left breast with associated vascularity (Fig. 1). A subsequent core needle biopsy of the lesion has shown this to be a benign MFB.

Following multidisciplinary team review, a decision was made to perform a wide local excision of the tumor. Subsequently, macroscopic histopathology revealed a well-circumscribed neoplasm surrounded by adipose tissue.

Microscopically, spindle-shaped myofibroblastic cells were observed to form haphazard fascicles separated by collagenous stroma. Occasional mild nuclear atypia was noted with one or two mitoses seen per 10 high-power fields (Fig. 2).

Although wide local excision was performed with a curative intent, the neoplasm abutted the anterior margin of the

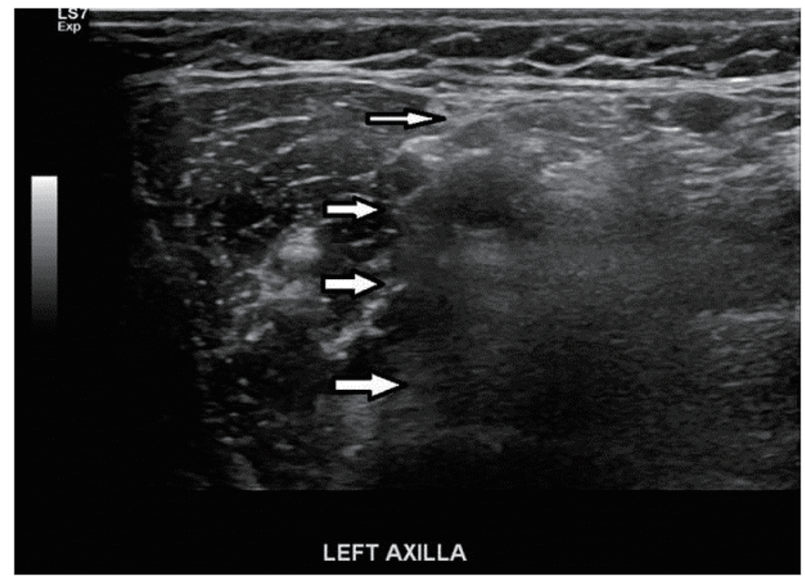

Figure 1. Ultrasonography showing $8 \times 3.8 \mathrm{~cm}$ round, well-defined, homogeneous mass (white arrows demonstrating the edges). 


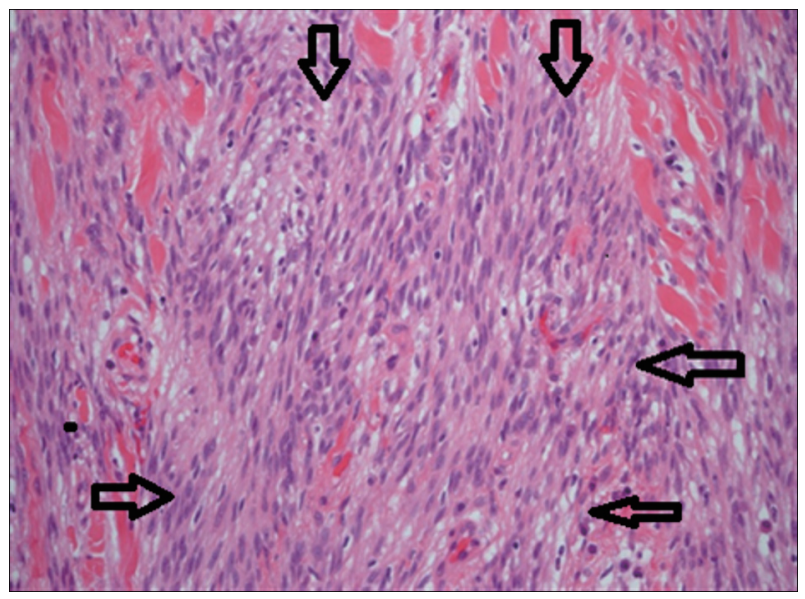

Figure 2. Spindle-shaped myofibroblastic cells forming fascicles separated by collagenous stroma.

resection, and therefore complete excision of the tumor could not be guaranteed. Follow-up ultrasound scans of breast at 3 and 8 months post-operatively were normal and there was no evidence of recurrence upon examination.

\section{Discussion}

Wargotz et al were the first to describe MFB in 1987. They reported 16 cases of this disease predominantly in men [2]. Since then, more than 70 cases of MFB were also reported in the English literature [4].

MFBs are rare benign mesenchymal tumors, arising from fibroblasts and smooth muscles. They belong to a family of benign spindle tumors of mammary stroma [5]. MFBs most commonly occur in the breast of men predominantly aged 25 - 87 years and post-menopausal women [5, 6]. In the existing literature, extra-mammary sites also have been reported.

The usual clinical presentation is a solitary, painless firm mass that is freely mobile. In our case, the mass was adherent to overlying skin, which is a unique feature of our case. As such tumors usually grow slowly over several months for up to $4 \mathrm{~cm}$; in our case the tumor size was larger at approximately $8 \times 3.8 \mathrm{~cm}$.

Histologically, MFB may exhibit several common pathological variants; however, these all exhibit common basic compositions and growth patterns. In our case, the histopathology examination showed a classical type MFB as described earlier.

Ultrasonography usually shows a well-circumscribed solid, homogenous, slightly hypoechoic mass. MFB often mimics fibroadenoma and therefore ultrasound alone cannot make a definitive diagnosis.

Clinical assessment and imaging alone may result in misdiagnosis of malignancy. Core biopsy is more appropriate test for diagnosis than fine needle aspiration $[7,8]$. MFB is usually diagnosed by histopathology with immunohistochemical staining showing immunoreactivity to CD34, desmin, smooth muscle actin and vimentin. However, sometimes MFB diagnosis might be challenging as it could be mistaken for a diagnosis of cancer in rare cases due to the presence of spindle cells [9]. MFBs are usually treated by surgical excision and no recurrences have been reported so far. In our case, the patient was monitored with regular follow-up scans up to 8 months and no signs of recurrence identified on scans as well as on physical examination. To our knowledge it is the first case to report adherence of the MFB to the skin which initially raised the possibility of malignancy.

The learning points are as follows: 1) MFB must be considered as a potential differential diagnosis in patients who present with a solitary painless breast mass; 2) MFB can present as mobile or fixed mass; 3 ) MFB often mimics fibroadenoma and therefore ultrasound alone cannot make a definitive diagnosis; and 4) Core biopsy is more appropriate for diagnosis of MFB than fine needle aspiration (FNA).

\section{Consent}

Written informed consent was obtained from the patient for publication of this case report and any accompanying images. A copy of the written consent is available for review by the editor-in-chief of this journal.

\section{Competing Interest}

The authors declare that they have no competing interest.

\section{Funding}

This article received no specific grant from any funding agency in the public, commercial, or not-for-profit sectors. None of the authors have any financial support or relationships that may pose a conflict of interest.

\section{References}

1. D'Ambrosio G, De Laurentis F, Scoglio D, Balla A, Quaresima S, Mattei F, Lezoche E. Breast myofibroblastoma in a young woman: a case report. Ann Ital Chir. 2013;84(ePub).

2. Wargotz ES, Weiss SW, Norris HJ. Myofibroblastoma of the breast. Sixteen cases of a distinctive benign mesenchymal tumor. Am J Surg Pathol. 1987;11(7):493-502.

3. Rovera F, Imbriglio G, Limonta G, Marelli M, La Rosa $\mathrm{S}$, Sessa F, Dionigi G, et al. Solitary fibrous tumor of the male breast: a case report and review of the literature. World J Surg Oncol. 2008;6:16.

4. Sharma A, Sen AK, Chaturvedi NK, Yadav R. Myofibroblastoma of male breast: a case report. Indian J Pathol Microbiol. 2007;50(2):326-328.

5. Magro G, Bisceglia M, Michal M, Eusebi V. Spindle cell lipoma-like tumor, solitary fibrous tumor and myofibroblastoma of the breast: a clinico-pathological analysis of 13 cases in favor of a unifying histogenetic concept. Vir- 
chows Arch. 2002;440(3):249-260.

6. Reis-Filho JS, Faoro LN, Gasparetto EL, Totsugui JT, Schmitt FC. Mammary epithelioid myofibroblastoma arising in bilateral gynecomastia: case report with immunohistochemical profile. Int J Surg Pathol. 2001;9(4):331-334.

7. Magro G. Mammary myofibroblastoma: a tumor with a wide morphologic spectrum. Arch Pathol Lab Med. 2008;132(11):1813-1820.
8. Powari M, Srinivasan R, Radotra BD. Myofibroblastoma of the male breast: a diagnostic problem on fine-needle aspiration cytology. Diagn Cytopathol. 2002;26(5):290293.

9. Kazani A, Mastoraki A, Dastamani C, Stafyla V, Kairi E, Psychogios I, Arkadopoulos N. Myofibroblastoma of the breast: diagnostic challenges of a rare benign tumor. Indian J Surg. 2015;77(Suppl 2):501-504. 